\title{
Towards Supporting Community Information Seeking and Use
}

\author{
Nkechi Nnadi \\ New Jersey Institute of Technology \\ nn5@njit.edu \\ Michael Gurstein \\ Centre for Community Informatics Research, Development and Training \\ gurstein@gmail.com
}

\begin{abstract}
In this paper we explore issues surrounding the design of systems that will effectively support community information seeking and use. We discuss the need for information retrieval systems to move away for the single user paradigm to one that recognizes the collaborative nature of information seeking and use. We also examine the collaborative information retrieval literature and derive implications for community informatics. The paper also explores the unique challenges of designing systems to support information seeking and use in a community context, and attempts to provide design guidelines that will enable researchers and practitioners to develop such systems.
\end{abstract}

\section{Introduction}

Information and communication technologies (ICTs) are widely believed to be a catalyst for both economic and social development. There is a prevailing sense that personal computers and access to the Internet are critical for economic success and individual improvement. However, in order to transform the lives and economies of people, particularly those in the developing world, ICTs must address their needs in a manner that goes beyond simply providing access to a wealth of technology, to providing a means of ensuring "effective use" of these technologies (Gurstein, 2003). Improved access alone is inadequate in realizing the transformative power of ICTs. Access alone cannot support economic development and social change. Instead effective use, which is defined as " the capacity and opportunity to successfully integrate ICTs into the accomplishment of self or collaboratively defined goals" (Gurstein, 2003), emphasizes the need for ICTs to work for people in their environment.

Information is seen as a key resource that can aid development (Meyer, 2004). Providing access to information alone is unlikely to empower communities and aid development. Information needs to be focused and targeted toward problems. Information provision will only lead to effective use when it is targeted toward issues such as new opportunities for improvement, markets and pricing, health information and valuable resources for improving productivity (McNamara, 2003). ICTs that support community information seeking and use may also have other beneficial side effects such as enabling quicker response by government agencies to communities needs, raising awareness of important issues, providing transparency in decision making processes and educating the community on environmental and market changes (McNamara, 2003). In order to harness the power of information for development, system designers must ensure that the collection, storage, retrieval and presentation of information is done in a manner that leads to effective use.

The traditional view of information seeking is one dominated by a focus on individual information seeking behavior. Many commonly used information retrieval (IR) systems, such as web search engines, assume that information seeking is carried out by individual users who describe their information need in the form of a query. This focus on the individual is arguably the result of classical models of information seeking behavior. One such model proposes that the individual develops an information need when he/she does not posses sufficient knowledge to complete a task at hand. This gap between what the user knows and what is needed to complete a task is called an anomalous state of knowledge (ASK) (Belkin, Oddy \& Brooks, 1982). In response to his/her anomalous state of knowledge, the user must seek out information that will bridge the gap in their knowledge and allow them to complete the task. The bridging of this gap is achieved through iterative query formulation and result evaluation, which is carried out until the individual absorbs enough information to complete their task. The ASK hypothesis posits that the need for information arises from the work tasks and daily activities of the user. However, work tasks are rarely solitary activities. Instead, organizations thrive by leveraging the combined abilities of many employees. Employees not only collaborate informally, but also are increasingly organized into teams that are expected to collaborate on tasks and activities.

It is important to recognize that the need for information arises from the collaborative activities of individuals. 
Information seeking and use can be seen as embedded in activities of workgroups rather than as a separate stand-alone activity. This view of information seeking and use is also applicable to communities. Communities may be organized informally around changing goals and tasks or may be more permanent with a long-term well-defined mission. In either case the success of the community depends on multiple individuals working together to achieve a common goal. It is reasonable to assume that in the course of working toward a goal a community will need to seek, create, share, store and manage information. The intrinsically collaborative nature of community tasks will require support from ICTs that are based on design principles that view information seeking and use as a collaborative and not a solitary activity. An explicit recognition of the collaborative nature of information seeking and use will have implications for designing community systems.

The traditional perspective of information retrieval systems also assumes the user can adequately represent their information need in the form of a query. This assumption is questionable. If we believe the ASK hypothesis that information need arise from knowledge gaps, then it is obvious that a user will not be able to adequately represent something he/she is unfamiliar with using a few keywords. Furthermore, the IR system's best match technique and resulting list of documents is thought to be a satisfactory means of solving the user's information need. This may not be the case especially if the user is illiterate or semi-literate. Thought must be given to designing systems for people from oral culture that may have very different conceptual structures (Meyer, 2003) and therefore do not conceive their information need in terms of a query that must be posed to a system.

Most information retrieval systems provide a list of documents in response to the query that is supplied by the user. However, this requires the user to go through the document list in order to discover useful information. This process is time-consuming and requires a lot of effort on the part of the user. In addition, a list of documents may also be inadequate if the user is first faced with the challenge of forming a clear idea of that information need. In this case the most important form of aid would be providing a process for defining the information need. Supporting the process of formulating the information need is very important but is usually overlooked or reduced to helping the user to choose query terms. A better solution, and one that would be helpful in facilitating community information seeking and use, would be to conceive the process of articulating the information need as one that is composed of discussion and consensus building. Defining an information need is essentially answering the question: what problem are we trying to solve? In some cases, this may involve reconciling differing and even conflicting viewpoints on an issue in order to arrive at a somewhat unified view of the problem. If the perspective of supporting problem formulation through consensus building is employed in the design of community systems, then features that allow discussion and consensus building such as discussion forums and voting tools must be tightly integrated to information storage and retrieval systems.

The importance of collaboration in information retrieval is gaining prominence and has been identified as an important area of research (Dumais, 2000). This increasing awareness of the social or collaborative nature of information retrieval may provide insights for the design of information systems for communities. All community endeavors are by nature collaborative because they involve a joint effort of individuals united by a common goal. In order to achieve its goals, communities must leverage the combined talents of its members. Information seeking in community informatics is one area that has not been well researched, however it is obvious that any information system that will support community information seeking must be built with collaboration in mind. In developing countries in particular, ICTs are likely to be shared among a number of people rather than individually owned (Prahalad \& Hammond, 2002). This is because aggregated purchasing power is more significant than the individual's purchasing power especially in countries with low per capita income. This implies that rather than the view of "one person, one computer", systems must be built with the assumption of "many people, one computer"; leading to the realization that both system use and community activity are collaborative. The collaborative nature of system use is an important consideration for the design of ICT's for community information seeking and use. This is because the location of the ICT becomes an important factor in determining if it will be used in the intended manner. If access to the ICT requires great effort on the part of the user, it is unlikely that it will become an integral part of the processes it was designed to support. Therefore, in designing community information systems, designers must also consider where the system should be situated in order to ensure effective use.

\section{A review of collaborative information retrieval studies}

Collaborative information retrieval is "any activity that collectively resolves an information problem" (Dumais et al, 2000, p. 298). In this sense information retrieval does not consist solely of query formulation and result evaluation, instead it includes the processes of problem identification, analysis of information needs, query formulation, retrieval interactions, and the presentation and analysis of results (Dumais et al, 2000). In addition it includes the processes by which IR activities are coordinated across multiple participants. This view of information seeking moves away from the solitary user metaphor. Instead it begins to examine the social aspects of information seeking and aims to support these social processes as well as the more technology-oriented challenges of building information retrieval systems.

Poltrock et al (2003) conducted a study of collaborative information seeking and sharing in design teams. They examined two teams of product designers in two different companies to determine the ways in which the team as a 
whole addresses its information needs. The first team was a software design team at Microsoft and the second a hardware design team at Boeing. The authors observed that collaborative IR was employed by the teams in order to understand product requirements, develop design specifications and analyze design alternatives. In the software design team, the team leader played a central role by coordinating work schedules and facilitating communications between the team and other departments or employees in the organization. The team met weekly at staff meeting, though much of the design work was done individually in private offices or in small subgroups of a few people. The need for information was often identified and discussed at the weekly meeting and when necessary, other Microsoft employees were consulted to satisfy the information need. Design specifications were shared with others in order to obtain feedback especially when product requirements were unclear. In contrast to the software design team, the hardware design team at Boeing was organized differently. Instead of individual offices they worked in an open office space. There was no strict division of labor. Team members rarely worked alone, instead the frequently consulted each other on design options and made decisions collaboratively. They consulted other employees in the organization especially those involved in other aspects of the development of the product they were designing.

Although both teams differed in their work habits, they had striking similarities in the manner in which they sought information. The members of both teams sought information about the product they were designing. The nature of the design process makes it difficult to express in clear terms what is needed at any point in time. The search for information is often the search for defining the information need. This activity is often done collaboratively. The activity is also directly related to the team member's existing task responsibility. It is also striking that the information need is not met by providing the right document even though both teams could conceivable have looked at specifications for similar products. Instead the information need was often resolved by consulting the right individual (or team) in the organization. The insights from this case study provide some guidance in designing information systems for communities. The collaborative way in which the information need is defined implies that system features that support discussion and consensus building are necessary. It reveals that providing contacts more than documents might be the best way to help a community solve its information needs.

Romano et al (1999) utilized a different approach to understanding collaborative information retrieval. They reviewed the literature on user experiences with information retrieval and group support systems (GSS), and also interviewed current users of information retrieval and group support systems (Romano, Russinov, Nunamaker \& Chen, 1999). Observations of user experiences reveal that IR users would like collaborative search capabilities. GSS were noted to be lacking in support for the collaborative use of information retrieved independently by group members during the course of group work. They also lack support for the use of retrieved information in decision-making and teamwork.

To address the single-user view of IR and GSS as well as their inadequate support for collaborative information seeking and sharing, the authors developed an integrated knowledge creation environment that combined features of GSS and IR systems. The system features included the ability for multiple users to share both queries and results, and the allowing team members to search collaboratively even though they were not physically located. Users were able to annotate query results, which allowed them to share their opinion of the validity of a particular document for the task they were trying to complete. The authors discovered that members the same group or team typically had different relevancy evaluations of documents. To promote consensus building the system was modified to provide electronic polling that allowed user's to rate an items relevance to the search task.

The case study highlights an issue that is often overlooked in the design of systems for communities. That is that the members of the community must decide the value of information jointly. The community's social dynamics must be considered when providing system features such as voting tools in order to promote consensus building or joint evaluation of information resources. For example, in traditional African societies elderly people are held in high esteem and their opinion is likely to affect the communities acceptance or evaluation of a particular piece of information (Meyer, 2003). Also in traditional cultures, there is a tendency toward strong hierarchical structures in the society that require decision making to be channeled through community leaders (Meyer, 2003). This means that the typical 'democratic' approach to providing systems that allow free and open dialogue may not be accepted in certain communities. Also the community might desire that information be sanctioned only by a select few rather than all members.

Another study of a software design team's collaborative information retrieval behavior was conducted by (Fidel et al, 2004). The authors defined collaborative information retrieval as "any event in which actors collaborated to resolve an information problem that required them to user resources external to their own knowledge". The software design team consisted of ten people with length of employment ranging from four months to seven years. Their study revealed that employees were motivated to search for information collaboratively, when the information need could not be met by any documents they had access to. The lack of documentation forced teams members to collectively define the information need, and determine potential information sources (usually people). The information retrieved was also evaluated and interpreted collaboratively, because various types of expertise possessed by the individuals on the team were needed to ensure that the entire team obtained a unified understanding of the information. A similar study was conducted by Hansen and Jarvelin (2005) in a Swedish patent office. Their study confirmed previous findings that collaborative activities centered on document creation and use as well as interpersonal communication and interaction 
between employees.

The case study reveals that the evaluation of retrieved results is done collaboratively in order to harness individual expertise. This is important for communities especially those that may be considered transient. This type of community organizes itself around a particular issue or common goal and once the goal is achieved the organization is no longer needed and may disperse to reorganize at a later time to address a new issue or goal. Due to the nature of these types of communities, its members bring a variety of different skills and levels of expertise. It is therefore important to direct a piece of information to the right individual for evaluation in order to harness that individual's talents. It may not be necessary that every member of the community assess a piece of information, but the information must be evaluated from all relevant viewpoints by those possessing the ability to determine its value.

Karamuftuoglu (1998) conceptualizes the problem of CIR differently. She believes that one reason that IR systems have been focused on the individual is because IR is viewed as a task of transferring knowledge from a database to users. In contrast collaborative IR should focus on the knowledge production function of IR systems. CIR should be based on cooperation between individuals that facilitates the creation of new knowledge from documents retrieved. She believes the production of new knowledge is typical in inventive tasks and is motivated by situations when the solution to the problem is not known in advance i.e. the user cannot retrieve a document that will satisfy the information need. This explanation seems to be supported by the previous studies of CIR in design teams. The design of a new product is a highly innovative and inventive activity. The novelty of the tasks constrains the users reliance on documents as primary sources of information. Instead, other people must be consulted in order to define both the problem and the solution. Karamuftuoglu's observation is notable because it provides insights to the relationship between collaborative information retrieval studies and designing systems to support community information seeking and use. Communities can be said to engage in multiple collaborative tasks, which are tasks that may not have a single clear goal, lack discrete start and end points, and in some cases may have incompatible goals (Johnson, May \& Johnson, 2003). These types of tasks require a high degree of coordination and collaboration. In addition, the indistinct nature of the goals requires the members of the community to be innovative and inventive in developing solutions. Therefore systems that will support community information seeking and use will have to consider how they facilitate the user in producing new knowledge not only from the collaboration between members but also from the collective evaluation and interpretation of information gathered from other sources.

\section{Challenges of Designing ICT's for Community Information Seeking and Use}

The challenge of supporting community information seeking and use goes beyond determining appropriate ICT features. The deeper issues lie in understanding how the community views its needs and how they perceive the role of ICTs in meeting those needs. To truly provide a tool that can be effective in supporting community information seeking and use one must consider socio-economic issues and provide answers to the following questions: How is information conceived in the community? In what manner should information be presented? In what location or during what occasions is information sought or shared in the community? What social interaction norms must be observed in the use of the system and how will this be accommodated in system design? Who will design, implement and maintain the system? Who are the individuals in the community that can participate in the design of the system? Whose support must be sought in the community in order to minimize resistance?

The questions raised above are not strictly design considerations but are essential to the successful development of any system that will support community information seeking and use. The question of how information is conceived in the community is one that many times is not considered. This is problematic because it ignores the fact that information and the knowledge derived from it are both social constructs that are grounded in a particular world view (Mchimbo, 2004). In his study of information use in rural development, Meyer noted that information is perceived differently in oral cultures than it is in literate ones. Oral cultures tend to utilize mnemonic patterns such as alliterations or proverbs to store information. They also tend to learn new information by relating it to concrete items in their world and tend to retrieve information by association (Meyer, 2003). Any system designed for a community must consider the form in which the community stores, shares and retrieves that information. This will affect the degree to which the community can utilize the system for effective use. It is also important to determine how the information will be presented once it is collected and stored. Metaphors that allow users to easily understand system functionality and associated information become crucial to the development of systems that will aid a community's information seeking and use. For example, the Cleveland Free-Net utilized a city metaphor to organize its information contents. Information related to a particular subject was placed in virtual buildings e.g. Supreme Court decisions could be found in the courthouse building (Schuler, 1994). Discovering local metaphors and patterns of information storage and sharing is crucial to the design of successful ICTs.

Not only is it important to understand the manner in which information is generated and represented in a community, but one must also understand the social norms that surround these processes. For example, a community's decision-making may traditionally be the function of community leaders. Any software that provides electronic voting as a means to reach a consensus on issues by all community members may undermine the influence of the community's elders and lead to friction in both the development and use of the system. The success of systems that support 
community information seeking and use hinges on the abilities of the system's designers to understand how social norms affect the creation, use and sharing of information. ICTs provide the unique ability to change the manner in which tasks are performed. It is the designer's responsibility to communicate the potential changes especially if they affect existing social norms and to obtain feedback from users before the implementation of the system.

Another important consideration is the location and manner in which information is typically shared in the community. In the collaborative information retrieval literature the behavior of the subjects is often observed in a work setting such as an office space or in a library. Communities, however, are not constrained by organizational processes that may provide some structure and predictability to information sharing. The manner in which they share information may vary based on the nature of the community or the type of task they are performing. The community may favor a collective forum such as a town hall meeting, or may share information in sub-groups during informal gatherings, or in the course of everyday activity. The issue of where a community shares information is also of importance. In developed countries, it can be assumed that community members own a personal computer or have access to one at a nearby public library. This means that information sharing via computer-mediated communication is a viable option and members of the community can express their information needs easily through a computer. However, in underdeveloped nations access to computers is limited to telecenters that may be many miles away. Therefore, there is a lag between the time at which the information need is discovered and when access to an ICT can be obtained to resolve this need. This makes it unlikely that the system will become an integral part of the community's activities. To be successful the system should be located in an "information ground".

An information ground is "an environment temporarily created by the behavior of people who have come together to perform a given task, but from which emerges a social atmosphere that fosters the spontaneous and serendipitous sharing of information" (Pettigrew, 1999, p. 811). The idea of an information ground emerged from Pettigrew's study of chiropody clinics. She observed that although the clinic was organized to provide foot care, the elderly patients utilized their time there to socialize and also obtain social service information from peers and clinic staff. The social atmosphere of the clinic fostered the sharing of information needs. The information need is not explicitly stated as it is in traditional information retrieval where the user must conceive of the information need in the form of a query. Instead, the information need emerges from casual social interaction. The information ground ceases to exist when the people have concluded their activities and leave. However, it comes alive again at the next gathering. ICT's for community information seeking should be situated in such places. This will allow them to be utilized when an information need is generated in the course of social interaction

In addition to determining the appropriate places to situate ICTs that will facilitate information seeking, system designers must also take into consideration the local context of use. Some researchers have advocated a change in focus when it comes to designing information systems for communities. Instead of focusing on the development of infrastructure, they advocate an understanding of the local conditions of the community (Salvador and Sherry, 2004). The key to developing ICTs to foster change is not in focusing on developing technological infrastructure, but rather in attaining a good understanding of the challenges the ICT must address and determining the manner in which ICTs can address these challenges (McNamara, 2003). The key to building a successful system to support community information seeking is to determine how to leverage whatever current infrastructure is available in order to meet the challenges facing the community. It is equally important to build flexible systems that allow for "local improvisation" that can accommodate the peculiarities of the system's context of use. Suchman (2002) views local improvisations as "the means by which anything — technological systems, organizational forms, everyday projects - are made to work" in a particular context that is different from the context in which it was developed (Suchman, 2002, p. 139). The lesson for ICT development then is to build transparent systems so that users can adapt the system to suit their needs after they have become familiar with it. Only through usage can local communities identify the requirements for a system that will support their information seeking and use. It is difficult to see how a community that is new to ICTs can completely inform the design of these systems before they have had any hands-on experiences with the new technology. Therefore, the implementation of a system is not an end in and of itself, but rather the opportunity to educate users and allow them to understand the possibilities of new technology.

A commonly suggested solution to ensuring that the system is designed to fit the local context of use is the utilization of a participatory design process. In this way, the end users are an active voice in the design of the system. They not only provide system requirements but also participate in design decisions. Although user participation is desirable, it is often difficult to implement a participatory design process when designing for communities in developing countries. Users may be unable to adequately express their desires for the system design, because they do not yet understand what the technology is capable of. Also finding the right people to involve is challenging. One way to combat this problem and incorporate user input would be through the identification and inclusion of information gatekeepers during the design process. Information gatekeepers are those individuals in the community who perform an official or unofficial role of connecting their community with resources needed to solve problems (Metoyer-Duran, 1991). The information gatekeepers often have significant community status and are able to interpret the community's information needs and broker information. They may also be early adopters of technology. These individuals are clearly able to provide useful information in the participatory design process. In addition to information gatekeepers, it is important to obtain the support of community leaders who are able to influence community attitudes to new information 
technology. Although these individuals may be unable to directly contribute to the design of the system, they are crucial in ensuring its adoption once the system is built. Mchumbo (2004) advocates the inclusion of as many community members as possible. However, he advocates that community participation not be limited to identifying information needs but so include development problems facing the community and the information needed to meet these challenges.

Although it may seem premature to discuss the maintenance of a system when design principles are still unclear, system maintenance is very important and ensures that the system continues to support the community's activities. System designers for community ICT's are typically imported from outside the community. In developing countries, system designers are hired by governmental or non-profit agencies, and are usually from developed countries (Heeks, 2002). The funding for ICT design and consequently the support for the designers is limited. It is natural that as time goes by there will be a need to resolve any bugs that may arise, and therefore a need for someone to resolve them. However, the maintenance of a system goes beyond just ensuring its continued functioning. As the community utilizes and becomes familiar with the system, they begin to identify the system's shortcomings as well as the potential for it to be modified to meet new needs. This point is illustrated by Sanderson and Fortin in their review of website development and use by community organizations in Canada (Sanderson \& Fortin, 2003). The authors observed that the initial website development is geared toward establishing a web presence for the community. It is only after the community has had the opportunity to interact with the new technology that they begin to incorporate it into the way they work, typically by modifying the system to support communication and developing content geared toward the local community. This means that a lasting solution would have to incorporate the education and training of local individuals. This has been recognized by certain governments that have made a concerted effort to promote the development of their information technology sector in order to provide skilled professionals (Akhtar \& Gregson, 2002).

\section{Guidelines for Designing ICTs to Support Community Information Seeking and Use}

This admittedly limited review of the studies of collaborative information seeking and the brief analysis of challenges unique to designing community systems provides some useful insights that can serve to inform the design of future systems. The first of these is that it is important to understand what form of information a community utilizes in exchanging ideas. Designers should not limit their vision to systems where text is the primary means of information exchange. Multimedia systems have the potential to allow non-literate communities to participate in using ICTs to facilitate their development, but their development may be limited by the lack of technological infrastructure. One way to get around this may be through the use of "old media" technologies such as radio and television to facilitate the exchange of information.

A second major theme emerging from the previous discussions is that collective evaluation of any information gathered by the community's members must be supported by the system. This means that system designers must pay attention to the inclusion of consensus building tools such as shared discussion spaces, moderated discussion threads, voting or polling features, and annotation features that allow users to express their views on the usefulness of a particular piece of information. Designers are cautioned however, to be mindful of the social norms that govern a community's information sharing and evaluation patterns. If there is a blatant conflict between the system design and established social norms, systems designers are encouraged to educate potential users on this consequence and try to win support for the design before the full implementation. In this way some user dissatisfaction and abandonment of the system can be avoided when system design clashes with the community's norms.

Another point that system designers must consider is building support for the use of the system by demonstrating its utility in combating pressing problems that the community faces. Promoting the systems ability to help the community target a concrete problem might be a good way of garnering support from community leaders who in some cultures are suspicious of technology. System designers must also seek the right type of people to participate in the development of the system, in particular by selecting the community's information gatekeepers. The gatekeepers will provide useful information regarding the information needs of the community and can also suggest system features that will enable the community to easily utilize the system.

Romano et al (1999) also indicate the need to provide support for real-time collaborative search. The ability to search with others across geographic boundaries is especially useful when one community needs to engage in a collaborative venture with another. In addition the authors address the needs for users to communicate their opinion about search results thus providing a means for not only information consumption but also information creation. Community members would benefit from not only sharing their opinions with each other, but also from the ability to store and retrieve these opinions for future use or dissemination.

Twidale and Davis (1996) echo these suggestions and provide a few more. The authors believe any comprehensive solution to collaborative information seeking should include a means to capture the user's search process. This will allow others to gain insights into the user's train of thought or provide suggestions at various stages of the search. They also believe that one way to facilitate community information seeking may be to provide matchmaking between people with similar interests. This is particularly useful in large communities that may have sub-groups with different interests. Lastly the authors advocate the facilitation of coordinated searches among dispersed users on remote terminals. The system should provide for a means of sharing screen displays of search results and communication in real time. 


\section{Conclusion}

This paper is an attempt to shed light on the challenges facing the design of systems that will effectively support community's information seeking and use. Much of the lessons for designing these community systems are derived from the study of collaborative information retrieval in workgroups. Although, communities face very different challenges than workgroups in organizations, some insights can still be garnered and used if one is mindful of the peculiar challenges of designing for community systems.

\section{References}

Akhtar, S. \& Gregson. J. (2001). Internet technologies in the Himalayas: lessons learned during the 1990s. Journal of Information Science 27(1), 9-17

Belkin, N. J., Oddy, R. N., \& Brooks, H. M. (1982). ASK Information Retrieval: Part 1. Background and theory. Journal of Documentation 38(2), 61-71.

Bruce, H., Fidel, R., Pejtersen, A. M. Dumais, S., Grundin. J., Poltrock, S. (2002). A comparison of collaborative information retrieval (CIR) behaviors of two design teams. Paper presented at the 17th IEEE International Symposium on Intelligent Control, ISIC'02, Vancouver, British Columbia, Canada.

Carroll, J. M. (1990). Infinite detail and emulation in an ontologically minimized HCI. Paper presented at the SIGCHI conference on Human factors in computing systems: Empowering people, Seattle, Washington, United States: ACM Press.

Dumais, S., Grundin, J., Poltrock, S., Bruce, H., Fidel, R., \& Pejtersen, A. M. (2000). Collaborative information retrieval. Paper presented at the Conference on Human Factors in Computing Systems, The Hague, The Netherlands: ACM Press.

Fidel, R., Pejtersen, A.M., Cleal, B., \& Bruce, H. (2004). A multidimensional approach to the study of humaninformation interaction: A case study of collaborative information retrieval. Journal of the American Society for Information Science, 55(11),939-953.

Gurstein, M. (2003). Effective use: A community informatics strategy beyond the digital divide. First Monday 8(12). Retrieved from http://firstmonday.org/issues/issue8 12/gurstein/

Hansen, P. \& Järvelin, K. (2005). Collaborative information retrieval in an information-intensive domain. Information Processing \& Management, 41(xxx): xxx-xxx, in press.

Heeks, R. (2002). Information Systems and Developing Countries: Failure, Success, and Local Improvisations. The Information Society, 18, 101-112.

Johnson, P., May, J. \& Johnson, H. (2003). Introduction to multiple and collaborative tasks. ACM Transactions on Computer-Human Interaction, 10(4), 277-280.

Mchombu, K., J. (2004). Sharing Knowledge Handbook 2. Retrieved Febuary 12, 2005, from http://www.oxfam.ca/publications/downloads/Sharing\%20Knowledge\%202\%20Inside\%20Pages.pdf

McNamara, K. S. (2003). Information and Communication Technologies, Povery and Develpment: Learning from Experience. Paper presented at InfoDev Annual Symposium, Geneva, Switzerland.

Metoyer-Duran, C. (1991). Information-seeking Behavior of Gatekeepers in Ethnolinguistic Communities: Overview of a Taxonomy. Libary and Information Science Research, 13, 319-346.

Meyer, H. (2003). Information use in rural development. The New Review of Information Behavior Research 4(1), 109125.

Meyer, H. (2005). The nature of information, and the effective user of information in rural development. Information Research, 10(2). Retrieved from http://informationr.net/ir/10-2/paper214.html

Pettigrew, K. E. (1999). Waiting for chiropody: contextual results from an ethnographic study of the information behavior among attendees at community clinics. Information Processing and Management, 35(6), 801-817.

Poltrock, S., Grundin, J., Dumais, S., Fidel, R., Bruce, H., \& Petjersen, A. M. (2003). Information Seeking and Sharing in Design Teams. Paper presented at the 2003 international ACM SIGGROUP conference on Supporting group work, Sanibel Island, Florida, USA: ACM Press

Prahalad, C. K. \& Hammond, A. (2002). What works: Serving the poor, profitably. Retrieved October 10, 2004, from http://www.digitaldividend.org/pdf/serving_profitably.pdf

Preece, J. (2000). Online communities: Designing Usability and Supporting Sociability. Chichester, UK, John Wiley \& sons. 
Romano, N. C., D. Roussinov, et al. (1999). Collaborative Information Retrieval Environment: Integration of Information Retrieval with Group Support Systems. Paper presented at the 32nd Hawaii International Conference on Systems Sciences, Hawaii, USA.

Salvador, T. and J. Sherry (2004). Local Learnings: An Essay on Designing to Facilitate Effective Use of ICTs. The Journal of Community Informatics 1(1). Retrieved from http://ci-journal.net/viewarticle.php?id=35

Sanderson, D. and A. Fortin (2003). Web Site Development by Locally-Oriented Community Organizations: Characteristics, Problems, and Issues. Paper presented at the Association for internet researchers 4.0, Toronto, Canada.

Shuler, D. (1994). Community Networks: Building a new participatory medium. Communications of the ACM, 37(1), 39-51.

Suchman, L. (2002). Practice-based design of Information Systems: Notes from the Hyperdeveloped World. The Information Society, 18, 139-144.

Twidale, M. B. and D. M. Nichols (1996). Interfaces to support collaboration in information retrieval. Paper presented at the BCS IR \& HCI Workshop, Glasgow, UK. 